\title{
RELEVANCE OF VASCULAR STRUCTURAL AND SMDOTH MUSCLE SENSITIVITY CHANGES IN HYPERTENSION
}

\author{
David F. Bohr, M.D. and Kathleen H. Berecek, R.N. \\ Department of Physiology, University of Michigan, Ann Arbor, Michigan 48109
}

\section{SUMMARY}

This is a mini-review of the vascular changes that occur in experimental hypertension. Augmented vascular resistance and reactivity appear to be due to an increase both in wall thickness and in vascular smooth muscle sensitivity. The increase in wall thickness is an adaptive change that is secondary to the increase in arterial pressure; the altered smooth muscle sensitivity occurs in the absence of an increase in arterial pressure and hence may initiate the increase in vascular resistance and reactivity of experimental hypertension. Studies of subcellular function indicate that changes in smooth muscle sensitivity may be caused by an increase in cell membrane permeability and an altered handling of calcium by its subcellular sequestering system.

Compared to the recent advances in the clinical management of the hypertensive patient, new insight into the basic pathophysiology responsible for this disease has been painfully deliberate. The physician now has effective hypotensive agents that will normalize blood pressure. Furthermore, it is known that once the pressure is normalized the risk factor for the morbid complications of hypertension has been greatly reduced. However, these agents only treat a symptom of the disease and do not deal with its cause. Mechanistically, although it is known that the increased arterial pressure is usually caused by an increase in total peripheral

This review was supported by U.S. Public Health Service grant HL-03756 from the National Heart and Lung Institute. K.H. Berecek is a predoctoral fellow supported by NIH grant PHS -4620 .

Address of Authors:

Department of Physiology

7710 Medical Science II

University of Michigan Medical School Ann Arbor, Michigan 48109

U.S.A. resistance, there is no comfortable consensus regarding the nature of the vascular changes that cause the increase in total peripheral resistance, nor has the sequence of extra-vascular events responsible for this increase been defined.

In approaching the broad problem of furthering our understanding of the vascular changes responsible for hypertension, two purely intellectual contributions can be made: 1) terms can be defined; 2) specific questions can be articulated.

Definition of terms:

1. Vascular resistance is a measure of the pressure drop across a vascular bed per unit flow. There is general agreement that it is elevated in nearly all types of hypertension.

2. Vascular reactivity is a measure of the increase in vascular resistance produced by a constrictor agent. Again there is agrement that this value increases in hypertension. Furthermore, it is generally recognized that the increase may be due to structural changes in the vessel wall or to functional changes in the vascular smooth muscle, or to both.

3. Vascular smooth muscle sensitivity describes the ease with which a vasoactive agent can cause contraction of vascular smooth muscle. It may be measured as the reciprocal of the concentration of vasoactive agent required to produce a threshold response. This response may be either the increase in Elow resistance through a vascular bed or the contraction of an isolated strip of vascular smooth muscle. However, since the flow resistance change is camplicated by possible differences in vessel wall thickness, a more critical evaluation of sensitivity can be made from the contraction of an isolated strip. It is difficult, however, to do these studies on smooth muscle from small resistance vessels. An increase in sensitivity will. also cause a parallel shift to the left of the concentration-response curve. The pro- 
blem of whether vascular smooth muscle sensitivity increases in hypertension cannot be thought of as being resolved.

4. Vascular smooth muscle contractility describes the maximum forcegenerating ability of vascular smooth muscle. It is best measured directly rather than in terms of altered flow resistance. Studies carried out on smooth muscie from large vessels indicate that its contractility is decreased in hypertension.

\section{What are the questions?:}

A. Structural changes in vessels

1. What is the nature of the structural changes?

2. Do they play a role in the development of hypertension?

3. Do they play a role in its maintenance?

B. Functional changes in vascular smooth muscle

1. Do such changes socur in hypertension?

2. Do they contribute to the cause or maintenance of hypertension?

3. What is their nature, defined at a subcel lular level?

4. What is their cause?

The principal objective of the following paragraphs is to sumarize the recent evidence bearing on functional changes that may occur in vascular smooth muscle in experimental hypertension. The summary of this evidence is preceded by reference to the structural changes in the vessel wall which must be recognized in studies evaluating possible functional differences.

Vessel wall structure:

Histologic studies $(1,2)$ have demonstrated that thickening of the vascular wall occurs with the development of experimental hypertension, and perfusion studies

(3) have clearly established that wall thickening can bring about increases in vascular resistance and reactivity. Folkow and his collaborators have demonstrated that an increase in wall thickness which encroaches on the lumen causes: 1) an elevated resistance of the fully dilated vascular bed, 2) a steep concentrationresponse curve to norepinephrine, and 3) an elevated maximum constrictor response.
Structural changes alone should not alter the threshold concentration of a vasoactive agent for the production of vasoconstriction. Observations made in both the spontaneously hypertensive rat (3) and the renal hypertensive rat (4) have met these criteria for the production of increased vascular reactivity by wall thickening alone without an increase in vascular smooth muscle sensitivity.

Recently Hutchins and Darnell (5) have described an entirely different structural basis for increased vascular resistance and reactivity. They found that the cremaster muscle of the genetically hypertensive rat had approximately half the normal number of small arterioles (12-25 u). They considered that this reduction in the number of arterioles might reflect a longterm autoregulatory reaction to overperfusion by the elevated arterial pressure. They have recently supported this hypothesis using hypotensive therapy to prevent the development of hypertension in this genetic strain. In these pharmacologically protected rats the decrease in the number of small arterioles did not occur.

Vascular smooth muscle sensitivity:

Same investigators $(3,4)$ have found no evidence for increased vascular smooth muscle sensitivity in hypertension. Others, using several experimental models of hypertension, various methods of study and sources of vessels have reported an increase in sensitivity. Same of these reports are listed in the Table. In most of these studies the evidence favoring an increase in sensitivity of vascular smooth muscle depends on the observation that the threshold of aqonist recuired for a contractile response or for an increase in resistance is lower in the hypertensive animal than in its nomotensive control. In addition there are three indirect types of evidence that support the view that an alteration in vascular smooth muscle sensitivity may be involved in the increased vascular reactivity of hypertension. These are: 1) An individuality in the increases in sensitivity of the vascular smooth muscle from the hypertensive animal depending on the constrictor agent used. If the increase in reactivity were due to wall thickening it would be expected that the response to all agonists would be equivalently affected. 2) Temporal relationships between the increase in vascular smooth muscle sensitivity and the increased blood 


\section{TABLE}

Recent studies presenting evidence in support of increased vascular smooth muscle sensitivity in hypertension.

Reference Numbers

Perfusion studies

$\frac{\text { Physiological salt }}{\text { solution }}$

Whole body

Mesentery

Hind quarter

Tail

9
$7,9,10$
9
32

Aucologous blood

Hind leg

17

Hind quarter

Critical opening pressure (rat tail)

Muscle bath preparations

$\begin{array}{ll}\text { Femoral artery } & 15,21 \\ \text { Aorta } & 8,31 \\ \text { Veins } & 18\end{array}$

pressure are compatible with the possibility that the increased sensitivity may cause the hypertension rather than be a result of it. 3) Increase in vascular smooth muscle sensitivity has been reported in the absence of the increase in wall stress of hypertension. It is seen in the venous system and in arteries and resistance vessels that have been protected from the elevated pressure, agair suggesting that the sensitivity change may be a generalized process of all vascular smooth muscle and not merely secondary to increased wall stress. The following paragraphs present details of these three types of evidence.

Individuality in sensitivity to several constrictor agents:

McGregor and Smirk (6) observed an increase in basal resistance and an increase in vaso-constrictor response to norepinephrine in the mesenteric arteries of both genetic and renal hypertensive rats. They found a much greater increase in responsiveness to 5-hydroxytryptamine than to norepinephrine. From this observation they introduced the argument that the increase in reactivity could not be due entirely to an increase in the wall-to- lumen ratio and that the differential augmentation of resistance produced by the two agonists indicates that there is a functional alteration in the smooth muscle of the resistance vessel in hypertension. This doservation of a greater responsiveness to 5-hydroxytryptamine than to norepinephrine was confirmed by Haeusler and Finch (7) in genetic, renal, and DOCA hypertensive rats. More recently another example of such a differential change in responsiveness has been observed both in the contraction of isolated strips of vascular smooth muscle (8) and in the resistance changes of perfused vessels (9). BY both methods the smooth muscle sensitivity in response to norepinephrine was greater in hypertension but there was no change in the response to calcium of potassium depolarized smooth muscle. Perhaps related to this difference is the observation by Collis and Alps (10), depicted in the figure.

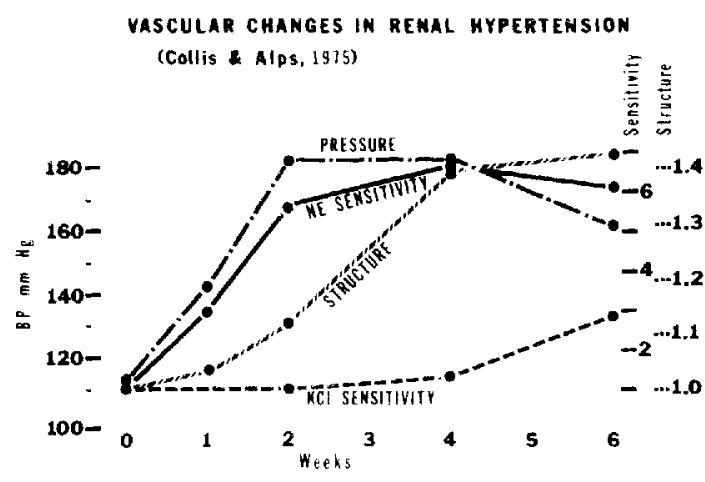

FIGURE:

Temporal development of various vascular changes following the initiation of renal hypertension in the rat. These values were derived from data presented by Collis and Alps (10). Sensitivity values are calculated as the dose ratio of a constrictor agent, control to hypertensive rat, required to produce a specific per cent of the maximum constrictor resporse of the mesenteric vascular bed. Structural differences are calculated as the ratio of the maximum pressor responses, hypertensive to control rat. Results were obtained from a constant flow perfusion of the mesenteric vasculature with a physiologic salt solution.

that the increase in sensitivity to norepinephrine in hypertension is much greater 
than that to $\mathrm{KCl}$. Also it has been observed that the augmentation of the constrictor effect of barium is less than that of norepinephrine (11). It has been suggested that the contraction caused by these cation manipulations may act more directly on the contractile component of the vascular smooth muscle and not involve an earlier event of the contractile sequence that is utilized by norepinephrine. This possibility leads to the suggestion that it is this earlier event in the contractile sequence, perhaps an initial membrane excitation, that is altered in hypertension. The difference between the increased sensitivity to norepinephrine and that to barium has been the basis of an interesting schematic model presented by Lais and Brody (11) which gives insight into the relative contribution of increased vascular smooth muscle sensitivity and increased wall thickness as determinants of the increase in vascular reactivity of hypertension.

Temporal relation between increase in smooth muscle sensitivity and development of hypertension:

The probability that a functional change in vascular smooth muscle is the cause of the increase in vascular reactivity and increase in vascular resistance in hypertension is supported if a functional change precedes or accompanies the development of hypertension. Jones $(12,13)$ observed a greater "leakiness" of the plasma membrane of the smooth muscle cell of SHR and DOCA hypertension. He observed this change in DCCA hypertension before the pressure rose (13). Collis and Alps (10) found that a rise in their index for vascular sensitivity to norepinephrine paralleled the rise in blood pressure in renal hypertensive rats (Figure). Whereas blood pressure and sensitivity were near maximm in two weeks their index of structural change had reached only about one-quarter of its maximum at this time. Shibata (14) observed that the abnormal sensitivity of SHR to non-physiological cations preceded the development of hypertension in these rats. Finch and Haeusler (9) found elevated arterial pressures and increased vascular reactivity in SHR 12 to 14 weeks old. There was no evidence of narrowing of the lumen at this age but by 28 weeks this also had occurred. Lundgren et al. (4) observed that the pressure rise in renal hypertension preceded evidence of a structural change. In sumary, the findings suggest that whereas functional changes in vascular smooth muscle precede or accompany the increase in arterial pressure and,hence may be causal, wall thickening develops later and is probably adaptive to increase in wall stress.

Increase in smooth muscle sensitivity in the absence of increase in wall stress:

Another approach that has been used to assess the role of altered vascular smooth muscle sensitivity in the aetiology of hypertension has been the protection of one vascular bed from the hypertension and observing the characteristics of a protected artery (15). Changes that occur in such an artery could not be secondary to increased wall stress and might be related to the cause of the increase in vascular resistance and reactivity. Chronic occlusion of one external iliac artery in the rat lowers the pressure in the occluded leg to approximately half of that in the contralateral unoccluded leg. It was found that the changes in femoral artery smooth muscle sensitivity associated with hypertension could not be reversed by lowering blood pressure in one leg of a spontaneously hypertensive rat or prevented by protecting one leg from high pressure prior to the induction of DOCA hypertension. This contrasts with the results of a similar hypotensive procedure in SHR in which a reversal of stmuctural changes in resistance vessels of the legs was observed by Folkow et a1. (16).

These contrasting observations might be interpreted as indicating that structural changes are secondary to an increase in wall stress whereas the increase in smooth muscle sensitivity is not. There is another interpretation: perhaps changes that occur in the small resistance vessels are pressure dependent whereas those occurring in the large femoral artery are not. In order to determine which of these interpretations is correct we have recently studied resistance vessel changes in hypertension (17) using our protectod leg technique (15). Instead of studying force development by a smooth muscle strip from a large conduit vessel, we observed vascular reactivity in the perfused hind limb. Hypertension was produced in the rat by slipping one renal artery into the 250 micron slit of a small silver block. The contralateral kidney was not removed. At the time of this surgery one extemal iliac artery was completely tied off. Four 
to six weeks after the development of a stable hypertension the vasculature of both hind Iimbs was studied with a bilateral blood perfusion system. Following complete relaxation of vascular smooth muscle with papaverine the flow resistance in the unprotected, high-pressure leg was 50 g greater than that in the protected leg indicating that the increase in structural resistance is secondary to the increase in pressure. There was no difference between the structural resistance in the protected leg and that in the unprotected leg of a normotensive rat. On the other hand, when the reactivities of these vascular beds to norepinephrine was studied it was found that the threshold dose required to produce a constrictor response in either the protected or the umprotected leg of the renal hypertensive rat was only about one-tenth as great as that required in the normotensive rat. Again it appears that although the structural changes may be secondary to the increase in wall stress there are functional differences in sensitivity that are independent of arterial pressure and, hence, may be related to the aetiology of this disease.

Recent observations that there are abnormalities on the venous side of the circulation $(18,19,20)$ add credence to the hypothesis that primary vascular changes occur unrelated to the increase in wall stress of arterial hypertension.

Vascular changes in different types of hypertension:

Altered sensitivity of the vascular smooth muscle cell is not similar in all types of hypertension. The increase in sensitivity to norepinephrine appears to be much more prominent in DOCA and renal hypertension than it does in SHR $(9,15,21)$. on the other hand, vascular smooth muscle from SHR is more sensitive to stimulation by strontium or lanthanum than is that from the other two types of experinental hypertension $(15,21)$. However, the structural changes in these three major forms of experimental hypertension seam to be similar except for the evidence that Finch and Haeusler (9) have presented that lumen narrowing occurs more slowly in SHR than in the other two.

Subcellular mechanisms for increased sensitivity:

A few observations have been made which give insight into the subcellular mechanisms that may be involved in the altered vascular smooth muscle sensitivity that occurs in hypertension. One of these observations is based on the fact that calcium in high ooncentrations depresses the vascular smooth muscle response (22). Presumably it binds to the plasma membrane thereby stabilizing it, reducing its excitability, and inhibiting contraction. Conversely, if the amount of calcium bound to the monbrane is low the stabilizing effect of calcium will be lessened and the membrane will be more sensitive to stimulation. We have observe? that compared to vascular smooth muscle from nomnotensive rats, that from SHR, DOCA, or renal hypertensive rats requires a higher calcium concentration to depress a contractile response $(15,21)$. This observation suggests that the plasma membrane of the vascular smooth muscle cell in hypertension behaves as if it has less than normal stability and that higher concentrations of calcium are requirea to produce this stabilization. Such a condition would exist if the menbrane in hypertension had fewer calcium binding sites or if the affinity for calcium of the existing sites were low. Jones $(12,13)$ has presented more direct evidence for this specific subcellular abnormality. He has observed that compared to vascular smooth muscle from the normal rat, that from the SHR or DOCA hypertensive rat has a plasma membrane which is "leakier" to potassium. "This leakiness is less reduced by increasing calcium concentration than is the normal (13) .

Friedman (23) has presented evidence indicating that the plasma membrane of the vascular smooth muscle cell in DOCA hypertension is leakier to sodium and potassium than is that fram the nomotensive control. Hermsmeyer (24) made interesting deductions regarding membrane properties in SHR fram electrophysiological studies of smooth muscle cells in the tail artery. These cells Erom SHR had a less negative membrane potential than those from control rats at $16 \mathrm{C}^{\circ}$, but not at $36^{\circ} \mathrm{C}$. He concluded that the plasma membrane was leakier to potassium in the hypertensive rat but that at physiological temperatures this was compensated by a more active Na-K electrogenic pump. He also observed that depolarization by norepinephrine was greater in the SHR than in the control, and postulatod that this could form a basis for the increase in vascular. smooth muscle sensitivity.

Studies on the subcelluiar micro- 
somal fractions of vascular smooth muscle from SHR $(25,26,27,28)$ indicate that calcium binding by this fraction (presumably plasma membrane and sarcoplasmic reticulum) is reduced compared to that from nomotensive control rats. These investigators have also observed an increase in calcium-dependent ATPase activity in microsames from SHR. The cambination of a low caicium uptake and an increase in ATPase activity may reflect a leaky membrane of the sarcoplasmic reticulum so that calcium cannot accumulate to a high level and an over-compensation of the ATP-dependent pump which sequesters calcium in the sarcoplasmic reticulum. Webb and Bhalla (26, 27) have also observed a reduction in $\mathrm{Cy}^{-}$ clic AMP binding sites in these microscmes from SHR. This deficit may interfere with the phosphorylation of the vesicular protein by protein kinase and membrane phosphoprotein phosphatase. Since vesicular calcium transport is regulated by the state of phosphorylation of vesicular protein, this deficit may be the basis for impaired calcium transport. Recently Mangelsen and Bohr have observed a similar decrease in the rate of calcium uptake by microscmal vesicles from renal hypertensive rabbits (29). Translated into terms of muscle function this impaired uptake of calcium by the sequestering system should increase the intracellular concentration of activator calcium, thereby increasing the sensitivity of the muscle to stimulation by a vasoconstrictor agent.

The information surveyed in this brief review indicates that it will be profitable to search further in this area for a more precise understanding of the nature of the vascular changes that cause the increase in total peripheral resistance in experimental hypertersion. In addition, for a complete definition of the pathogenesis of this disease it will be necessary to identify the sequence of events by which the initiating intervention (e.g., renal artery constriction or DOCA implantation) produces these vascular changes.

\section{REFERENCES}

1. HATT, P.-Y. (1972): Electron microscopic study of arterial lesions in experimental hypertension. In Hypertension ' 72 ( $\mathrm{J}$. Genest and $\mathrm{E}$. Koiw') 196-2.12 (Springer-verlag, Berlinf Heidelberg).
2. TODD, M.F., and FRIEDMAN, S.M. (1972): The ultrastructure of peripheral arterios during the development of DocA hypertension in the rat, z. Zellforsch, 128,538 .

3. Folkow, B. (1971): The haemodynamic consequences of adaptive structural changes of the resistance vessels in hypertension, clin. Sci., 4l, I.

4. LUNDGREN, Y., HALLBACK, M., WEISS, L., and Folkow, B. (1974): Rate and extent of adaptive cardiovascular changes in rats during experimental renal hypertension, Acta. Physiol. Scand., 91, 103.

5. HUTCHINS, P.M., and DARNELL, A.E. (1974): Observation of a decreased number of small arterioles in spontaneously hypertensive rats, Circ. Res. 34 and 35 , Suppl.1, 161.

6. MCGREGOR, D.D., and SMIRK, F.H. (1970): vascular responses to 5-hydroxytryptamine in genetic and renal hypertensive rats, Am. J. Physiol., 219, 687.

7. HAEUSLER, G., and FINCH, L. (1972): vascular reactivity to 5-hydroxytryptainine and hypertension in the rat, Arch. Exptl. Pathol. Pharmakol, 272, 101.

8. FIEID, F.P., JANIS, R.A., and TRIGGIP, D.J. (1972): Aortic reactivity of rats with genetic and experimental renal hypertension, Can. J. Physiol. Pharmacol., 50, 1072 .

9. FINCH, L., and HAEUSLER, G. (1974): Vascular resistance and reactivity in hypertensive rats, Blood Vessels, Il, 145.

10. COLIIS, M.G., and ALES, B.J. (1975): Vascular reactivity to noradrenaline, potassium chloride, and angiotensin II in the rat perfused mesenteric vasculature preparation, during the development of renal hypertension, Cardiovase. Res. , 9. 118

11. LAIS, L.T., and BRODY, M.J. (1975): Mechanism of vascular hyper-responsiveness in the spontancously hypertensive rat, Circ. Res., $36 \& 37$ suppl. 1, 216.

12. JONES, A.W. (1973): Altered ion transport in vascular smooth muscle from spontaneously hypertensive rats, Circ. Res., 33, 563.

13. JONES, A.W., and HART, R.G. (1975): Altered ion transport in aortic smooth muscle during deoxycorticosterone 
acetate hypertension in the rat, Circ. Res., $37,333$.

14. SHIBATA, S., KURAHASHI, K., and KUCHII, M. (1373): Fossible etiology of contractile imoairment of vascular smooth muscle from spontaneously hypertensive rats, J. Pharmacol. Exp. Ther., 185, 406.

15. HANSEN, T.R., and BOHR, D.F. (1975): Hypertension, transmural pressure, and vascular smooth muscle response in rats, Circ. Res. , 36, 590.

16. FOLKOW, B., GUREVICH, M., HAILBACK, M., LUNDGREN, Y., and WEISS, I.: The hemodynamic consequences of regional hypotension in spontaneously hypertensive and normotensive rats, Acta. Physiol. Scand., 83, 532 .

17. BERECEK, K.H., and BOHR, D.F. (in press): Bases for increased vascular reactivity in experimental hypertension, Proc. 2nd Int'I. Symp. Vasc. Neuro-effector Mechanisms (5. Karger, Basel).

18. BEVAN, J.A., BEVAN, R.D., PEGRAM, B.I., PURDY, R.E., and SU, C. (1974): Increased responsiveness of veins to adrenergic stimulation in experimental. hypertension, Blood Vessels, 11, 241.

19. GREENBERG, s., and BOHR, D.F. (1975): Venous smooth muscle in hypertension: Enhanced contractility of portal veins from spontaneously hypertensive rats, Cire. Res. $36 \& 37$, Supp1. 1, 208.

20. SIMON, G., PAMNANI, M.B., DUNKEL, J.F., and OVERBECK, H.W. (1975): Mesenteric hemodynamics in early experimental renal hypertension in dogs, Circ. Res., 36,791 .

21. HOLLOWAY, E.T., and BOHR, D.F. (1973): Reactivity of vascular smooth muscle in hypertensive rats, Circ. Res., 33 , 678.

22. BOHR, D.F. (1963): Vascular smooth muscle: Dual effect of calcium, Science, 139, 597.

23. FRIEDMAN, S.M. (1974): An ion exchange approach to the problem of intracellular sodium in the hypertensive process, Circ. Res. 34, Suppl-1, 123.

24. HERMSMEYER, K. (in press, 1976): Electrocgenesis of increased norepinephrine sensitivity of arterial vascular smooth muscle in hypertension, Circ. Res.
25. AOKI, K., YAMASHITA, K., TOMITA, N., TAZUMI, K., and HOTTA, K. (1974): ATPase activity and $\mathrm{Ca}^{++}$binaind ability of cubcellular membrane of arterial smooth muscle in spontaneously hypertensive rat, Jap. Heart J., 15 , 180.

26. WEBE, R.C., and BHAILA, R.C. (1975): The role of cyclic AMP and calcium in vascular smooth musclo of spontaneously hypertensive rats (SHR), gnat. Record, $181,543$.

27. WEBB, R.C., and BHALIA, R.C. (in press, 1976): Altered calcium sequestration by subcellular fractions of vascular smooth muscle from spontaneously hypertensive rats, I. Mole. Cell. Caraiol.

28. MOORE, I., HURWITZ, L., DAVENPORT, G.R., and LANDON, E.J. (1975): Energydependent calcium uptake activity of microsomes from the aorta of normal and hypertensive rats, Biochim. et Biophys. Acta, 413, 432 .

29. MANGELSEN, E.I., and BOHR, D.F. (in press, 1976): Calcium handling by the microsomal fraction of aorta from the hypertensive rabbit, Physiologist.

30. DARKE, A.C., and GASKELL, P. (1973): A contrast in vascular reactivity between two kinds of experimental hypertension studied by measurements of the critical opening pressure of tail vessels in the conscious rat, Bib1. Anat., 12, 429.

31. GROLLMAN, A., and KRISHNAMURTY, V.S.R. (1973): Contractile response of the aorta of the nomotensive and acute and chronic hypertensive rat, Arch. Int. Pharmacodyn. Ther., 203, 375.

32. BEILIN, I.J., WADE, D.N., HONOUR, A.J., and COLE, T.J. (1970): Vascular hyper-reactivity with sodium loading and with desoxycorticosterone induced hypertension in the rat, clin. Sci., $39,793$. 


\section{DISCUSSION}

DR. FOLKOW: From Dr. Bohr's experiments and from a study in Fleidelberg which was presented at the fourth meeting of the International society of Hypertension in the Opera House, we have heard that in e.g. corticosteroid hypertension there is a true super-sensitivity response of the smooth muscle elements. Looking at the SHR rats, it seems to be quite aifferent in our experience there is no evidence of any true super-sensitivity to, for example, noradrenaline. On the other hand, the contractile elements seem to differ in some other respects and Dr. Bohr showed one example with strontium etc; we have observed that the very smallest arterioles and the venous side of the vascular bed (which are not exposed to increased pressure) maintain their contractility much better in the sHR rat than in the normotensive rat at low calcium levels. I have no doubt something happens on the functional level too, and of course, it is very important to look at both the functional and the structural elements and to keep these aspects together, as they are very much interwoven.

DR. BROD: Dr. Bohr, ten years ago you presented some fascinating data, in Cleveland I think, about the difference in the reactivity of renal and muscular vessels to angiotensin; you found that in vitro the renal vessels are the least reactive and the muscular vessels most reactive, whereas in vivo it was the opposite. Have you further ideas on the cause of this difference? I would also like to say that from a clinical point of view the extremity vessels are least sensitive to the effect of blood pressure in that the development of atherosclerosis of these vessels doesn't seem to be more frequent in hypertensives than in normotensives. I should like to ask whether it wouldn't be a good idea to use a technique similar to the one you have used to examine e.g. the renal or coronary vessels, which in hypertensives are much more prone to structural changes.

DR. BOHR: You are interested in the angiotensin response we observed in renal arteries - some 500 microns O.D. in the dog; they respond poorly to angiotensin, in contrast to e.g. rabbit aorta, and rat portal veini the characteristic of a poor-responding vessel, e.g., dog coronary artery, is that it responds poorly in the isolated bath. The relationship between

\section{CHAIRMAN: DR. NYBERG}

good and poor responders in the isolated bath seems to depend on how rapidly they become tachyphylactic. I feel that whatever the tachyphylactic mechanism to angiotensin, it is great in the renal artery of the dog. On the question of individuality of vascular smooth muscle from different sites, I think there is a goldmine here for studies just because they are so different in response to various agonists. I don't know the answer to your question.

DR. HUNYOR: Could I just ask Dr. Bohr whether he has taken any smooth muscles or vessel pieces from untreated essential hypertensives and hung them up in a bath as Dr. Folkow alluded, and has he found, as Dr. Folkow implied, that there is no increase in sensitivity of these smooth muscle cells in the isolated organ bath?

DR. BOHR: Three different students of mine have done this over the past five years. We have a surgeon who delights in giving us pieces of bleeding vessels he finds during laparotonies. The variability from vessel to vessel among normals has always been so great that I am not satisfied that there is any difference. I have not been able to show a difference between normal subjects and hypertensive patients.

DR. HOH: I am most intrigued by Dr. Bohr's data on the differential sensitivity of the various smooth muscle strips from the various animals with regard to calcium or strontium, or lanthanum. This raises the interesting possibility that the difference might lie in troponin within the smooth muscle, because troponin, as you all know, is the regulatory protein in linking excitation to contraction in skeletal, cardiac smooth muscles, and some other contractile systems. It is known that troponin from skeletal and cardiac muscle differs in sensitivity to calcium and strontium, therefore finding this sort of difference might suggest that troponin is somehow different. So I'd like to ask whether the difference shown is observed in spontaneously hypertensive rats as well as in renal hypertensive rats and whether you had this troponin in mind when you did the experiment.

DR. BOHR: I run into a problem with this question because troponin was, up to two 
years ago, the regulator protein for mammalian smooth muscle. About five years ago myosin turned out to be the regulator protein for mollusc muscles, and in the last two years the myosin molecule has turned out to be the regulator protein for chicken gizzard and mammalian vascular smooth muscle. Your question is still good. I don't know exactly what causes this difference in the response to nonphysiologic cations. I really feel that it is much more superficial: cxcitation contraction coupling. Maybe the membrane doesn't handle these cations, or handies them differentiy. Then the differences that have been seen between vascular smooth muscle at a sub-cellular level may prove to be in the plasma memorane rather than in the chemo-mechanical transducing system and its regulation. Is there any relevance at all to the fact that these bivalent cations and lanthanum will contract the vascular smooth muscle from the genetic hypertensive rat? Is there any relationship to hypertension? The answer, very dogmatically, is that it is unknown. I can certainly imagine that there could be a relationship. Manganese, for instance, is present in all rats and all humans. Maybe smooth muscle is responding to manganese. I don't know of any genetic study that has been carried out to link hypertension to this peculiar characteristic of the smooth muscle.

DR. SIMPSON: we have been doing a number of perfusion experiments in the regional vascular beds, and so we are very interested in this field. One point that arjses is the difference, which we find anyway, if you use physiological saline and blood. We were interested to find in a perfused mesenteric artery preparation, a greatiy increased reactivity to noradrenalino and to 5-hydroxytryptamine in the saline perfused preparation in our strain of geneticaliy hypcrtensive rats, but no such increased reactivity in normotensive rats. We investigated this and it seemed to $\mathrm{lic}$, as I think Dr. Bohr mentioned, in the cell membrane. By use of verapamil and calciumfree solutions, and potassium-depolarised perfusate one was able to abolish the difference between genetically hypertensive and normotensive rats. So we presumed that the difference lay in the calcium handing at the cell membrane. However, the problem is that when you use blood perfusion, this difference between the genetically hypertensive and normotensive rats seems to disappear. So we don't know really whether this is of any relevance in the intact preparation. I just wonder whether Dr. Bohr finds this kind of difference between blood perfusion and saline perfusion?

DR. BOHR: When we compare our studies on blood perfused hind legs with our studies in the isolated bath, that gap is bridged and they are quite similar. The sensitivity to the non-physiologic cations is the same in both places. 